\title{
Emergency medicine in South Africa - time to catch up
}

The recent development of emergency medicine in South Africa (SA) provides a shining example of how much can be achieved by so few when passion and vision are combined with a commitment to improving patient care. Maturation of the specialty in countries such as Australia has embedded the role of emergency physicians in clinical niches where it would seem unnatural, inappropriate and even unsafe to place doctors with alternative training. As British and Australian emergency physicians who recently enjoyed the privilege of a study tour around SA, we were impressed and bewildered by aspects of SA's emergency care. We observed care in 6 hospitals and 2 pre-hospital systems in 3 major cities and a rural town. We offer our perspectives, not as experts or superiors, but as outsiders free from political allegiance or local institutional affiliation.

The case load of blunt and penetrating trauma in SA dwarfs that of other English-speaking nations, resulting in impressive specialist expertise. We had privileged contact with surgeons who ranked among the best in the world. However, in some areas, the apparent uniquely SA division between trauma and non-trauma emergency units within the same hospital made little sense to those of us used to the efficiency of a single emergency department. Resource implications aside, we were most struck - even in some very large and relatively well-equipped hospitals - by the absence of trauma care aspects considered mandatory in an emergency unit, e.g. timely provision of analgesia, minimum monitoring standards for procedural sedation and rapid sequence intubation, structured triage, allowing relatives access to patients undergoing resuscitation, the use of ultrasound for rapid diagnosis of life-threatening pathology, and supervision of junior staff. Ironically, emergency physicians in SA already have policies and recommendations in place for these issues, ${ }^{1-5}$ but are unlikely to see them enforced in departments that are not covered by emergency physicians.

SA emergency medicine is ahead of the game in pre-hospital and retrieval medicine; in other non-mainland European countries, we struggle to interest our emergency medicine colleagues in this practice to the point of having to create a new subspecialty. ${ }^{6}$ Pre-hospital care in SA seems well-integrated into the emergency medicine curriculum, with several areas seeing deployment of physicians to critically ill or injured pre-hospital cases. There is clearly a spectrum of critical care competence within the levels of ambulance personnel in SA, and, in some cases, it appears that the inter-hospital transport of critically ill patients might benefit from closer involvement of emergency physicians.

Our visit to a rural referral centre revealed what was possible when trained emergency physicians take responsibility for developing a comprehensive emergency medicine service that encompasses traumatic, medical and surgical emergencies. Obvious limitations in material resources and staffing did not prevent the provision of skilled resuscitation, point-of-care sonography, emergency departmentbased anaesthesia, procedural sedation, rapid turnaround minor surgery, orthopaedic lists, critical care (the need for which is heightened by limited intensive care beds), skilled acute medicine and toxicology, and, most strikingly, an emphasis on patient comfort and dignity. It is little surprise that such a department is regarded as a beacon for the acquisition of resuscitation and emergency procedural and anaesthesia skills by overseas graduates who value the structured training and senior supervision by trained emergency physicians. ${ }^{7}$

Despite only 7 years' existence as a registered specialty in $\mathrm{SA},{ }^{8}$ the training of emergency medicine registrars demonstrates state-of-the- art educational principles delivered by a faculty whose enthusiasm, commitment and clinical credibility would challenge that found in any longer-established overseas programme.

Arguments favouring the employment of emergency physicians in resource-limited settings include safety, governance and clinical versatility. As more of the population become educated, insured and medicolegally aware, many traditional attitudes and approaches will become obsolete and unacceptable. Resuscitation expertise aside, the role of the emergency physician as patient advocate in a resource-limited health system would occupy a void in many of the environments that we witnessed.

Seven years into official recognition, a disconnect remains between the skills and aspirations of emergency physicians and service provision in many areas. The websites of the two main public hospitals in the nation's capital exclude emergency medicine from their list of services; ${ }^{9} 10$ this is a revelation that this disconnect cannot be blamed purely on resources, but perhaps also on politics and culture.

Shortly after returning to Australia, SA was included in a wellpublicised World Health Organization report demonstrating that most preventable patient deaths were caused by inadequate clinical staff training and supervision, and failure to follow policies or protocols. ${ }^{11}$ This seemed to echo our observations that emergency and trauma units lacked emergency physician coverage.

We are inspired by the specialty's progress in such a short time, but much work remains. The first generations of emergency medicine graduates will need to be excellent clinicians, leaders, lobbyists, negotiators and managers. ${ }^{12}$ The challenge for other specialists, healthcare managers and politicians is to recognise that emergency medicine offers an economically sound solution to many practical, clinical and ethical problems in the care of ill and injured patients in SA. It is time to catch up.

Cliff Reid, Karel Habig, Ruby Hsu, Sarah Coombes

Staff specialist emergency physicians

Ambulance Service New South Wales

Sydney, Australia

\section{Corresponding author: C Reid (reidcg@me.com)}

1. Stander M, Wallis LA; Emergency Medicine Society of South Africa. Procedural sedation in the emergency centre. S Afr Med J 2011;101(3):195-201.

. Emergency Medicine Society of South Africa. Guideline: EM010 - Verification of endotracheal tube placement. http://emssa.org.za/practice-guidelines (accessed 23 March 2012).

3. Emergency Medicine Society of South Africa. Guideline: EM014 - Implementation of the south african triage scale. http://emssa.org.za/practice-guidelines (accessed 23 March 2012).

4. Gordon ED, Kramer E, Couper I, Brysiewicz P. Family-witnessed resuscitation in emergency departments: Doctors' attitudes and practices. S Afr Med J 2011;101(10):765-767

Bruijns SR, Engelbrecht D, Lubinga W, Wells M, Wallis LA. Penetrating the acoustic shadowsEmergency ultrasound in South African emergency departments. S Afr Med J 2008;98(12):932-934.

6. Emergency Medicine Subspecialty: Pre-hospital Emergency Medicine. http://www.gmc-uk.org/ education/emergency_medicine.asp (accessed 23 March 2012)

Smith ZA. Emergency and aeromedicine in Zululand. BMJ Careers, 2011

8. Balfour C. Emergency medicine - a new era in South African medicine. S Afr Med J 2006;96(1):47-48

9. Chris Hani Baragwanath Hospital. http://www.chrishanibaragwanathhospital.co.za/departments (accessed 23 March 2012). 10. Johannesburg Hospital. http://www.johannesburghospital.org.za/departments/departments.html
(accessed 23 March 2012).

11. Wilson RM, Michel P, Olsen S, Gibberd RW, et al. Patient safety in developing countries: retrospective estimation of scale and nature of harm to patients in hospital. BMJ 2012;344:e832. [http://dx.doi. org/10.1136/bmj.e832]

12. Bruijns SR, Wallis LA. Emergency medicine, an opportunity to re-imagine a speciality in Africa. Afr J EM 2011;1(2):49-50.

S Afr Med J 2012;102(11):836. DOI:10.7196/SAMJ.5913 